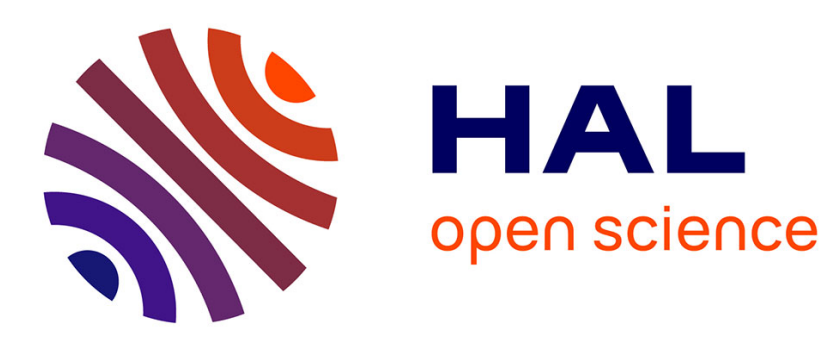

\title{
La construction détachée à gauche dans l'alternance des tours de parole
}

\author{
Lidia Likhacheva
}

\section{To cite this version:}

Lidia Likhacheva. La construction détachée à gauche dans l'alternance des tours de parole. Journal of French Language Studies, 2010, 10.1017/S095926950999038X . hal-02465386

\section{HAL Id: hal-02465386 \\ https://hal.science/hal-02465386}

Submitted on 3 Feb 2020

HAL is a multi-disciplinary open access archive for the deposit and dissemination of scientific research documents, whether they are published or not. The documents may come from teaching and research institutions in France or abroad, or from public or private research centers.
L'archive ouverte pluridisciplinaire HAL, est destinée au dépôt et à la diffusion de documents scientifiques de niveau recherche, publiés ou non, émanant des établissements d'enseignement et de recherche français ou étrangers, des laboratoires publics ou privés. 


\title{
La construction détachée à gauche dans l'alternance des tours de parole
}

\author{
LIDIA LIKHACHEVA \\ CLLE-ERSS, CNRS UMR5263, Université de Toulouse Le Mirail
}

\begin{abstract}
Dans le présent article, je me propose de montrer comment les fonctions discursives de la Construction Détachée à Gauche (CDG) liées au marquage de la structure informationnelle topique/commentaire se réalisent dans des contextes interactifs. Je pose notamment que la façon dont les occurrences de la CDG sont distribuées à travers la succession de tours de parole dépend en partie d'une stratégie discursive déployée par le locuteur, à savoir l'élicitation ou la narration. Les faits présentés ici ont pour visée de fournir des éléments de réponses nouveaux qui aident à mieux comprendre le rôle de la CDG dans l'organisation thématique et séquentielle du discours.
\end{abstract}

\section{INTRODUCTION}

Il est généralement admis que les exemples comme ceux qui suivent contiennent des occurrences de construction détachée à gauche (CDG):

(1) GS: Alors, je dirais que c'est quand même assez rare qu'une classe pendant cinquante ans ait de l'activité. Parce que des voyages on en a fait pas mal. (42ags1)

(2) MA1: Mes amis ça fait quand même une dizaine d'années que je les ai. (21ama1g)

(3) CD: Mes frères euh, maman était sévère avec eux aussi, mais un petit peu moins. Elle leur laissait la permission de sortir. (42acd1g)

Malgré la simplicité apparente du phénomène en jeu, son étude ne va pas sans difficultés: une littérature abondante en témoigne bien (Ashby, 1988; Barnes, 1985; Berthoud, 1996; Gregory et Michaelis, 2001; Lambrecht, 1981, 1994; Larsson, 1979; Pekarek Doehler, 2001; Prince, 1997; Ziv, 1994).

Dans la définition de la CDG, de nombreux auteurs mettent en avant le placement d'un constituant, le plus souvent un syntagme nominal, en position détachée à gauche et sa reprise anaphorique et/ou coréférentielle au sein de la proposition immédiatement suivante. Malgré ces quelques convergences, la description des propriétés syntaxiques et pragmatiques des CDG posent plusieurs problèmes. 
Pour commencer, la position extra-prédicationnelle et l'indépendance syntaxique du segment détaché à gauche impliquent que ce dernier se caractérise, en quelque sorte, par un statut 'hors syntaxe'. En effet, de nombreux syntacticiens se mettent d'accord pour reconnaitre que les difficultés liées à la description syntaxique de la CDG viennent du fait que le rapport entre le segment détaché et la proposition adjacente n'est pas de l'ordre de la micro-syntaxe (ou morphosyntaxe), mais de la macro-syntaxe (ou pragmasyntaxe).

Pour ce qui est des propriétés pragmatico-informationnelles de la CDG, il semblerait que la fonction prototypique de la CDG soit le marquage intonosyntaxique de la relation topique/commentaire où le topique est encodé par le constituant détaché à gauche, et le commentaire par la proposition qui le suit immédiatement (Barnes, 1985: 9). Le fait que la CDG permet d'expliciter ce qui sera le topique de la proposition qui suit et de respecter ainsi le 'Principe de Séparation de Référence et de Rôle" (qui veut que l'on ne puisse pas amener à l'existence discursive un référent nouveau et apporter en même temps de l'information à son propos) a conduit certains auteurs à poser que la fonction principale de la CDG est l'introduction et la promotion du référent sur l'échelle de l'acceptabilité topicale du statut non actif au statut actif (Geluykens, 1992; Givón, 1983; Gregory et Michaelis, 2001 (supra); Gundel, 1988a, b; Hidalgo, 2002; Lambrecht, 1994, 2001).

Cela étant, le fait que l'opération de détachement à gauche peut porter non seulement sur un référent nouveau, c'est-à-dire un référent qui n'a pas encore été établi dans le discours en tant que topique, mais aussi sur un référent qui a déjà accédé au statut de topique, a conduit certains auteurs non seulement à mettre en doute la primauté de la fonction introductrice de la CDG, mais à rejeter l'analyse informationnelle de la CDG en terme du marquage de la relation topique/commentaire (cf. De Fornel, 1988; Pekarek Doehler, 2001; Prince, 1997).

En partant du constat que les approches d'inspiration pragmatique négligent quelque peu l'apport interactif de la CDG dans l'échange conversationnel (voir à ce sujet les critiques de De Fornel 1988 et de Pekarek Doehler 2001), je me propose, dans ce qui suit, de montrer sur l'exemple de la CDG comment un discours peut associer, d'une part, les stratégies discursives qui relèvent de la gestion des topiques, et d'autre part, la configuration séquentielle de la conversation.

\section{LES DONNEES}

Les analyses du français, présentées ici, sont validées sur deux points d'enquête, Dijon et Roanne, réalisés dans le cadre du projet 'Phonologie du

\footnotetext{
1 'Do not introduce a referent and talk about it in the same clause' (Lambrecht, 1994: 185).
} 
Français Contemporain (PFC): usages, varietés et structure'2. Les points d'enquête Dijon-Roanne incluent 17 locuteurs, dont 8 hommes et 9 femmes, issus de milieux socio-économiques divers: employés du privé, fonctionnaires, professions libérales (par exemple, traducteur 'freelance', intermittent du spectacle, graphiste...), artisans ou ouvriers. L'ensemble des entretiens compte environ 70,000 mots. Les exemples tirés du corpus PFC sont identifiés grâce au code spécial ${ }^{3}$ (voir les exemples ci-dessus).

Selon le protocole adopté dans PFC, le transcripteur ramène les diverses réalisations d'un mot à sa forme standard ${ }^{4}$. Chaque locuteur est désigné par ses initiales: ' $A B$ ' ou ' $L C$, etc. Les initiales sont suivies de deux points (:). L'enquêteur est désigné par ' $E$ '. La ponctuation utilisée dans les annotations est quelque peu simplifiée. Une virgule marque une pause brève ou un contour intonatif de continuation. Un point marque une pause longue ou une fin d'énoncé qui est marquée mélodiquement. Une question est indiquée par un point d'interrogation. Ne sont utilisés ni le point d'exclamation ni les points de suspension.

Le changement de tour de parole est signalé par le changement de locuteur et le retour à la ligne. Si l'intervention d'un deuxième ou d'un troisième locuteur ne se traduit pas par une véritable prise de parole entrainnant un changement d'énonciateur, elle sera indiquée entre chevrons à l'intérieur du tour de parole du premier locuteur. Les mots tronqués sont indiqués par une barre oblique suivie d'un espace. Le discours rapporté est signalé par des guillemets simples (',') en début et en fin de discours.

\section{LA CDG ET L'ORGANISATION SEQUENTIELLE DE LA CONVERSATION}

S'agissant de la distribution des CDG dans l'alternance des tours de parole, on peut constater que la fonction introductrice de la CDG se réalise non seulement en début de tour de parole mais également à l'intérieur ou vers la fin du tour. L'analyse des entretiens guidés et des conversations libres montre que l'introduction du topique ne s'attache pas à une étape particulière dans l'organisation séquentielle du discours. Toutefois, il semblerait que l'introduction du topique dans une CDG soit incompatible avec le début absolu de la conversation. D'ailleurs, les corpus analysés ne contiennent aucune

2 PFC est un projet international, axé sur l'étude descriptive et comparative de la prononciation du français contemporain (voir à ce sujet Durand, Lyche et Laks, 2002; Durand et Lyche, 2003). Les informations sur le projet sont disponibles sur le site PFC: http://www.projetpfc.net/.

${ }^{3}$ Afin de respecter l'anonymat des locuteurs et l'indexation opératoire des fichiers au sein de projet PFC, il a été proposé d'utiliser un code spécial comportant les informations relatives à l'enquête elle-même et au locuteur (supra).

${ }^{4}$ Les motivations qui expliquent le choix de ce type de transcription sont exposées dans Durand et Lyche (2003) et Durand et Tarrier (2006). 
occurrence de la CDG en début de conversation. Ceci ne me semble pas étonnant dans la mesure où la position détachée à gauche accepte difficilement les référents à statut entièrement nouveau (cf. le statut 'brand new' de Prince 1981). Selon certains auteurs, ce rejet est justifié par l'inacceptabilité des exemples comme celui qui suit:

(4) *Un article de Robert Jackson, je l'ai lu hier. (Charolles, 2002: 152)

Ces observations sont confirmées partiellement par nos données de corpus: le SN détaché est préférentiellement exprimé par un SN défini, nom propre ou pronom disjoint. Ceci dit, contrairement à ce que l'on pourrait croire, l'indéfini spécifique n'est point exclu du site du détachement à gauche:

(5) AD: Un beau jour, je me rends compte, je dis, un gars $\mathbf{i l ~ m e ~ d i t ~ ' A h ~ b e n ~ d i s , ~ t i e n s ~}$ j'ai eu une promotion.' 'Oh là là.' j'ai dit. Au beau jour, je vais trouver le chef et je dis 'Alors euh, dites donc ma promotion.' 'Ah ben', il dit, 'vous êtes le deuxième sur la liste.' (42aad1)

Comme on peut le constater, il est possible que le locuteur introduise un référent nouveau dont l'allocutaire n'a pas d'expérience intersubjective ou discursive préalable. Toutefois, force est de constater que ce type d'emplois est très rare (dans notre corpus, 3 occurrences sur 250), ce qui prouve, me semblet-il, que la position détachée à gauche accepte difficilement les indéfinis spécifiques.

Le fait que l'introduction du topique ne s'attache pas à une étape particulière dans l'organisation séquentielle de la conversation ne signifie pourtant pas qu'il n'y ait pas de corrélation entre les fonctions discursives de la CDG et les contraintes d'ordre interactionnel qui régissent la conversation.

Tout échange conversationnel a une organisation interne bien complexe. Les séquences qui entrent dans sa composition pourraient être définies à partir des entités qui régissent thématiquement le discours. De telles entités sont des topiques, ou des macro-topiques, selon le cas. En effet, à un moment ou à un autre, le discours s'articule autour d'un topique particulier. De ce fait, le temps que le topique reste au centre de l'attention des interlocuteurs délimite en quelque sorte les frontières entre les différents segments de discours. En même temps, l'organisation thématique du discours doit être clairement distinguée de l'organisation séquentielle de la conversation.

Par organisation séquentielle, les analystes de la conversation entendent une combinatoire hétérogène de tours de parole qui se veut cohérente sur le plan relationnel, pragmatique et cognitif. L'alternance de tours de parole est liée par définition au changement de locuteurs. Toutefois, il se peut que le moment où finit un tour de parole et commence un autre soit difficile à établir à cause des entassements ou chevauchements entre les différents tours, ce qui n'est pas sans compliquer l'étude de l'interaction conversationnelle. Par exemple: 
(6) AD: Et alors euh. Toutes les fois euh, au mois de, arrivé le mois d'octobre pas, je m'étais pas méfié au début. Euh, arrivé le mois d'octobre euh, 'Ben tenez dites donc euh, pff. Faudrait que vous alliez à tel endroit euh.' Service bon, <CD: Ah oui il changeait de service. $>$ alors j'y allais, et puis arrivé à la fin de l'année, pas de promotion. (42aad1)

Le premier constat que l'on peut faire est que la construction du topique dans le discours ne relève pas d'une stratégie discursive unique. Rappelons qu'un référent de discours se voit attribuer le statut de topique lorsqu'il fonctionne comme support d'information dans une relation d'à-propos ${ }^{5}$. Cette définition ne dit pourtant rien sur le pourquoi de l'introduction d'un topique dans le discours. Or, comprendre les raisons sous-jacentes qui motivent l'introduction d'un topique est important pour l'approche interactionnelle de la topicalité car la façon dont procède le locuteur dépend directement de ses intentions communicationnelles. Plus particulièrement, le choix d'une stratégie discursive particulière dépend de ce que l'on veut obtenir par l'introduction d'un topique.

Pourquoi le locuteur introduit-il un topique? La réponse à cette question ne va pas de soi. Toutefois, il est possible d'identifier deux motivations majeures, à savoir: la présence ou l'absence d'information à propos de ce qui nous intéresse. En effet, on peut introduire le topique dans le discours parce qu'on détient de l'information à son propos et veut la partager avec les autres, ou bien parce qu'on manque d'information et espère en obtenir des autres. Quoi qu'il en soit, l'introduction d'un topique est fonction de l'intérêt que lui porte le locuteur (Chafe, 1994: 121).

De façon générale, ces deux visées communicationnelles se traduisent dans le discours par deux stratégies discursives différentes: l'élicitation et la narration (Chafe, 1994: 122-132). La différence entre les deux stratégies se situe principalement au niveau séquentiel. Lorsque la construction du topique se fait selon le schéma de l'élicitation, le discours repose sur la contribution alternée de deux ou plusieurs interactants. Les rôles des interlocuteurs diffèrent toutefois en ce qui concerne l'apport d'information: quoi que ce soit le locuteur ('eliciter' dans la terminologie de Chafe) qui choisisse et introduise le topique, seuls ses interlocuteurs ('responders') sont en mesure de satisfaire son besoin d'information. Ce format de l'interaction implique par définition que le topique soit préférentiellement relancé par celui qui y porte un vrai intérêt. Observons:

(7) E: Mais tu, en fait ouais ton, ton boulot de lectrice là, tu continues donc et. MB: Ah ouais, ouais, ouais.

E: C'est $[\ldots]$ un gros boulot ou euh?

MB: Oui, et puis c'est super mal payé donc euh.

E: Mais c'est vraiment, tu as beaucoup euh, beaucoup de trucs à rendre euh ouais? MB: Ouais, ouais, ben surtout, enfin là, plus trop mais euh, la maison d'édition pour Plon là, alors eux euh. C'est vraiment un rythme euh. (21 amb1)

${ }^{5}$ Le topique est entendu ici au sens de Lambrecht (1994). 
La construction du topique à travers la narration est quelque peu différente. Pour faire avancer le topique, le locuteur n'a pas besoin de la contribution alternée des interlocuteurs. Par contre, ce dont il a besoin, c'est leur attention. Ainsi, le succès du développement narratif du topique dépend également, quoique à moindre degré, de l'intérêt qu'il suscite chez les allocutaires. Selon Chafe, l'intérêt des interlocuteurs est acquis dans le cas où l'apport d'information à propos du topique comprend des éléments d'information nouvelle et inattendue qui entrent en conflit avec les attentes des allocutaires. Pour éclairer ce propos, examinons l'exemple ci-dessous:

(8) CD: Et puis voilà, donc le premier soir on a couché à l'hôtel, parce que l'appartement qu'on avait réservé n'était pas libre, donc on a couché à l'hôtel. Alors l'hôtel, les bagages, ils voulaient pas nos bagages, c'était pas l'heure. Alors que normalement, c'était à onze heures du matin, ils nous ont dit: 'Non, à cinq heures de l'après-midi.' On a dit: 'Mais non, vous plaisantez.' Alors, quand même ils ont accepté de les mettre dans une petite pièce, là-bas au fond. Donc on était débarrassés des bagages, on a pu un petit peu marcher plus tranquillement. Donc le soir, on a dormi à l'hôtel, et le lendemain, on a téléphoné à la propriétaire de euh, la location donc on a trouvé l'appartement. (42acd1)

Ayant postulé que la construction du topique peut se faire selon l'élicitation ou la narration, on pourrait supposer que les propriétés distributionnelles de la CDG sont liées directement au choix de l'une de ces deux stratégies discursives. Dans la narration, la CDG serait préférentiellement utilisée à l'intérieur du tour de parole, tandis que, dans l'élicitation, les occurrences de la CDG surviendraient plutôt au début ou à la fin du tour, plus particulièrement dans une question ou dans une réponse en réaction.

\subsection{La CDG et la construction du topique par élicitation}

Pour comprendre comment les tours de parole s'enchaînent entre eux, on devrait se munir d'un modèle d'agencement séquentiel de base qui soit applicable à la plupart des tours de parole. Selon Sacks (1987: 55), un tel modèle existe et se présente sous forme d'une paire adjacente de deux tours de parole: 'The basic sequence is a two-unit sequence; the two turns in which the parts of the sequence occur are placed adjacently to each other; and for all of them you can discriminate what we call "first pair parts" from what we call 'second pair parts', so that the parts are relatively ordered' (Sacks, 1987: 55).

La paire adjacente de deux tours de parole se laisse caractériser par au moins deux propriétés. La première correspond à la présence d'un lien entre deux tours de parole. Pour Sacks, les informations fournies dans le deuxième tour de parole doivent remplir les conditions de pertinence pragmatique que projette le tour précédent. De ce point de vue, le premier tour de la paire adjacente régit pragmatiquement le deuxième tour au sens où le type et la nature du premier tour projettent un nombre restreint d'alternatives quant au type et la nature du deuxième tour. Par exemple, la question sélectionne nécessairement une 
réponse, une salutation sélectionne une salutation, un compliment sélectionne un remerciement, et ainsi de suite (Sacks, 1987: 56). Pour se rendre compte du statut particulier du premier tour, il suffit de voir que les déviations par rapport aux attentes projetées par le premier tour sont perçues comme pragmatiquement inappropriées.

La projection d'une continuation préférentielle par le premier tour de parole n'implique pourtant pas que le deuxième tour ne puisse pas se transformer en premier tour d'une nouvelle paire adjacente:

(9) Comment vas-tu?
B: Je vais bien. Et toi?

La deuxième propriété de la paire adjacente de tours de parole découle logiquement de la première. Pour être pertinent sur le plan pragmatique, le deuxième tour de parole doit s'accorder préférentiellement avec la ligne thématique du tour précédent. C'est-à-dire, si le locuteur $A$ introduit un topique dans une question, le locuteur $B$ doit répondre en tenant compte du choix thématique de son interlocuteur, ce qui explique que les premiers éléments d'information fournis dans la réponse sont nécessairement mis au compte du topique introduit dans le tour précédent.

Si l'on examine la fonction introductrice de la CDG en rapport avec la succession des paires adjacentes de tours de parole, on peut constater que le changement du topique vs. l'introduction d'un nouveau topique ne peut pas se produire au début du deuxième tour de parole, surtout lorsque le tour précédent contient une question. Les données des corpus PFC confirment également cette observation. Lorsque le locuteur utilise une CDG dans le deuxième tour de parole, il le fait de façon à respecter les conditions de pertinence pragmatique projetées par le tour précédent: son intervention constitue une réponse en réaction qui s'accorde thématiquement avec le tour précédent:

(10) E: Et vous avez rapporté beaucoup de souvenirs? Non, du euh, des objets, des cadeaux?

GS: Oh ben des objets des cadeaux euh, il y en euh, il y en a eu pas mal. Parce qu'entre tous euh. Des tapis quoi, bien entendu des tapis. Euh b/, beaucoup de tapis je crois, il y a eu beaucoup de tapis, puis deux trois ba/, bibelots par ci là. Ah mais des tapis parce qu'on s'est arrêté à euh. Dans une fabrique. Qu'on a été reçus là à euh. (42ags1l)

S'agissant d'une paire adjacente de deux tours de parole, on pourrait supposer que le locuteur tienne à montrer à son interlocuteur que le référent que celui-ci vient d'introduire a bien été installé dans son modèle de discours et qu'il a l'intention d'en faire le topique de son intervention (cf. Lambrecht, 1981: 63). D'ailleurs, le statut intermédiaire d'un tel référent se reconnait au fait qu'il est exprimé par un SN à tête nominale et non pas un pronom. 
Selon Lambrecht (1981: 63-64), la reprise du référent dans une CDG au début du deuxième tour de parole se ferait dans le souci d'établir 'some sort of communicative agreement between the speech participants". Ainsi, la CDG permet non seulement de faire accéder le référent au statut de topique, mais également de réaliser la fonction phatique du langage qui relève du caractère social de la communication. La CDG peut en effet être considérée comme l'un des moyens de la réalisation de ce que Gramley et Pätzold appellent 'bearer support maxim (2003: 168) qui veut que, dans l'interaction conversationnelle, le locuteur poursuive le double objectif: il est censé non seulement enrichir la conversation sur le plan informationnel, mais il doit faciliter et encourager le contact avec le récepteur, c'est-à-dire se monter intéressé, attentif et coopératif.

En comparaison de la réponse avec une CDG au début du deuxième tour de parole, la réponse directe et univoque, sans la mobilisation explicite du référent négocié par l'interlocuteur, témoigne plutôt d'un moindre intérêt pour le choix thématique de l'interlocuteur.

A titre d'illustration, comparons l'exemple (10) avec l'exemple ci-dessous:

(11) E: Mais euh, ta, ta maîtrise de lettres, ça portait sur quoi?

MB: L'écriture des camps de concentration, les témoignages euh, sur Marguerite Duras et Robert (XX). Ça n'a rien à voir avec l'anglais.

E: Et l'Angleterre, enfin le, pour Erasmus là, c'était la seule destination où euh? MB: Oui, j'aurais préféré partir en Irlande mais, il y a des, des échanges très précis euh, la fac de lettres euh, c'est euh, Manchester, c'est tout quoi. (21 amb1g)

En (10), l'enquêteur introduit deux candidats au rôle de topique 'souvenirs' et 'objets et cadeaux'. Les deux apparaissent en position d'objet, choisie préférentiellement pour l'introduction d'un référent à faible degré d'accessibilité référentielle (cf. la position détachée à gauche). La question illustre ici l'un des principes qui régissent l'interaction conversationnelle, à savoir le Principe de Préférence pour l'Accord (Sacks, 1987: 58). Selon Sacks (op. cit.: 63), le premier locuteur formule sa question en sorte d'obtenir non seulement une continuation préférentielle pour le second tour de parole mais aussi un accord'. Les deux questions de l'enquêteur sont du type 'oui ou non'. Le fait que le locuteur cherche à ce que son interlocuteur réponde par un 'oui' se manifeste à travers la reformulation de la deuxième question. N'ayant pas reçu de réponse à sa première question, il y répond lui-même par un 'non' et reformule ensuite la question en essayant d'anticiper sur ce que pourrait être la bonne réponse (pour l'analyse d'un exemple anglais du même type, voir Sacks,

${ }^{6}$ 'By elevating the referent to topic status, the speaker wishes to express something like the following notion: "You want to know something about the $\mathrm{X}$ in question? $\mathrm{OK}$, let's talk about X. X is..."' (Lambrecht, 1981: 63).

${ }^{7}$ Dans les données d'usage analysées, il a observé notamment qu'en réponse à une question du type 'oui ou non', les locuteurs répondent plus fréquemment par un oui que par un non. Toutefois, si le locuteur formule une réponse «en désaccord' avec la continuation préférentielle projetée par la question, il tend à adoucir son désaccord en le repoussant vers la fin de l'intervention (Sacks, 1987: 57; de Fornel, 1988: 112). 
op. cit:: 64). La réponse qui suit dans le deuxième tour respecte non seulement le Principe de Préférence pour l'Accord mais aussi le Principe de Contiguité (op. cit:: 58). D'une part, le locuteur confirme la suggestion formulée dans la question, et d'autre part, il fournit une réponse qui s'ancre explicitement au tour précédent par le truchement de la reprise littérale des SN introduits dans la dernière question. Cet exemple met en évidence un autre fait intéressant. Le référent repris en position détachée à gauche au début du deuxième tour reçoit un développement topical ultérieur, quoique de façon spécifique - par le biais de l'introduction des référents sémantiquement dépendants 'des tapis' et 'des bibelots'.

En (11), la question est une proposition ouverte en X. La condition de pertinence pragmatique y est également respectée, mais de façon minimale. La locutrice $M B$ ne fait que fixer la valeur de la variable (Présupposition de E: la maîtrise de lettres de $M B$ portait sur $X$ ). Au niveau informationnel, le premier énoncé du deuxième tour de parole a la structure informationnelle du type topique/commentaire au sens où la variable 'L'écriture des camps de concentration, les témoignages, sur Marguerite Duras et Robert (XX)' constitue un apport d'information à propos du topique 'la maîtrise de lettres de $M B^{\prime}$ qui reste ici implicite. Il est à noter que la deuxième phase de l'établissement interactif du topique y est absente. En effet, la deuxième étape est une étape pragmatiquement importante, mais elle n'est pas obligatoire, surtout si le référent introduit dans la question est présumé accessible et tenu pour acquis par l'interlocuteur. Dans ce sens, la question projette non seulement une continuation préférentielle pour la réponse, elle projette également les instructions interprétatives quant à l'identifiabilité du référent dénoté par l'expression nominale. En (11), l'utilisation de l'expression référentielle ta maitrise de lettres et sa reprise immédiate par le pronom ça véhiculent une présupposition existentielle d'unicité au sens de Charolles (2002).

Le fait que la CDG n'est pas utilisée systématiquement au début du deuxième tour de la paire adjacente s'explique, à mon avis, par 'the Maxim of Quantity' de Grice (1975):

Q1: Make your contribution as informative as is required (for the current purposes of the exchange).

Q2: Do not make your contribution more informative than is required.

En effet, la réponse contenant une CDG est pragmatiquement plus coûteuse que la réponse contenant uniquement la partie informative de l'énoncé. A ce propos, l'exemple (11) présente une très bonne illustration de la maxime en question. Non seulement la locutrice $M B$ ne mobilise pas explicitement le topique de la question, mais elle bloque en quelque sorte le développement ultérieur de ce topique. Son manque d'intérêt pour le topique ne passe pas d'ailleurs inaperçu. Ayant attendu sans résultat que la réponse soit complétée, l'enquêteur change de topique et pose une nouvelle question. Dès lors, on peut conclure que l'étendue du topique dans le deuxième tour de 
parole de la paire adjacente est fonction des intentions communicationnelles du locuteur. Si le locuteur n'a pas l'intention de se réapproprier le topique, il est fort probable qu'il se contente juste de fournir la variable sur laquelle porte la question, comme dans l'exemple (11).

La comparaison des exemples (10) et (11) met en évidence une autre particularité intéressante. La façon dont le candidat au rôle de topique est introduit dans la question dépend de son accessibilité informationnelle. L'expression dénotant un référent à accessibilité informationnelle faible ou nulle ne peut apparaitre en position détachée à gauche mais tend à être placée en position d'objet. Par contre, le référent à statut activé, nouveau ancré ou familier (cf. Gundel et al, 1993: 274-307; Prince, 1981: 237) peut être introduit sous forme de syntagme détaché (cf. exemple (11)). Cette différence de statut informationnel se manifeste également dans l'organisation topicale du deuxième tour de la paire adjacente. En (10), le référent 'des objets et des cadeaux' accède au statut de topique seulement dans le deuxième tour de parole lors de sa reprise par le pronom en. A l'opposé, en (11), le référent 'la maitrise de lettres de MB' accède au statut de topique dans la question. D'ailleurs, ce fait pourrait expliquer pourquoi la locutrice $M B$ ne reprend pas le référent en question dans son tour de parole. Se trouvant au foyer de l'attention immédiate de deux interlocuteurs, le référent n'a pas besoin d'être marqué explicitement pour préserver son statut de topique actif.

Quelle est donc la visée pragmatique de la reprise du référent en position détachée à gauche par le deuxième locuteur? En effet, la comparaison des exemples (10) et (11) montre que la réponse peut être faite sans la mobilisation explicite du topique introduit dans le tour précédent.

L'explication de ce fait est à chercher, me semble-t-il, non pas dans l'organisation informationnelle ou thématique de la conversation mais plutôt dans l'organisation stratégique au sens de Roulet (2001). Dans ce qui précède, j'ai souligné à plusieurs reprises que, dans l'interaction conversationnelle, l'avancement du topique ne dépend pas seulement de la volonté de la personne qui parle. Le rôle des allocutaires est très important, surtout si la construction du topique se fait selon le schéma d'élicitation. Dans cette optique, la réponse fournie dans la deuxième partie de la paire adjacente est révélatrice en ce qui concerne la façon dont le choix thématique du premier locuteur est accueilli par le deuxième locuteur. En effet, bien que la réponse du deuxième locuteur doive remplir les conditions de pertinence pragmatique projetées par le premier tour, le locuteur a une certaine marge de manœuvre. Il peut se limiter à une réponse concise qui contient juste la partie focale de la réponse ou se réapproprier le choix thématique de son interlocuteur. Si, dans le premier cas, le topique négocié est un topique 'par défaut', dans le deuxième cas, il est un topique d'adoption'.

Le fait que le deuxième tour subit une contrainte de continuation préférentielle projetée par le tour précédent n'implique pourtant pas que le changement du topique soit impossible dans le deuxième tour. Seulement, le changement ou la réorientation thématique ne se fait pas dans le début absolu 
du tour de parole, surtout si le deuxième tour constitue une réponse en réaction. En effet, si le locuteur veut introduire un autre topique, il le fait après une réponse minimale préférentiellement sélectionnée par le tour précédent.

Ayant relevé certaines particularités du fonctionnement de la CDG au début du deuxième tour de parole de la paire adjacente, il s'agira à présent de décrire les propriétés qui caractérisent l'utilisation de la CDG au premier tour de la paire adjacente du type question.

En partant du principe que le premier constituant de la paire adjacente projette une continuation préférentielle pour le deuxième constituant, on pourrait supposer que la question avec une CDG permette au locuteur de remplir une double fonction communicationnelle: d'une part d'annoncer l'introduction d'un topique, et d'autre part, de mettre en évidence l'intérêt pour son propre choix thématique au sens où, si on introduit un topique, c'est parce qu'on le trouve intéressant.

Sur le plan informationnel, la question avec une CDG se voit attribuer la structure informationnelle topique/focus, le focus correspondant ici à la requête d'information. Pour ce qui est de la réponse, nous l'avons vu, elle doit remplir, même minimalement, les conditions de pertinence pragmatique et thématique projetée par la question.

Essayons maintenant de voir à quelles étapes dans la construction interactive du topique correspond l'utilisation de la CDG.

N'étant pas liée par la contrainte de continuation préférentielle, la première partie de la paire adjacente devrait permettre au locuteur d'introduire un topique nouveau ou de réintroduire (relancer) un topique désactivé (cf. Morel 1989: 158-159). Dans les deux cas, la question avec l'utilisation d'une CDG ne peut être cohérente, me semble-t-il, que, si elle constitue une déviation par rapport au tour précédent. Qu'en est-il en réalité?

Dans les données analysées, le recours à la CDG dans une question constitue $10,8 \%$ de cas (sur le total de $250^{8}$ occurrences de CDG), dont $25,92 \%$ servent à introduire un topique nouveau. Par exemple:

(Sowvenirs du Maroc)

GS: Alors on arrive, on euh, bien entendu, on demande du vin. Alors le premier coup, c'était à Ouarzazate. On demande du vin et il y en avait. Bon. Il y en avait, on boit bon, on boit chacun une bouteille, mais quand on a voulu en redemander, il y n'en avait plus. Parce qu'ils avaient épuisé le stock. Puis alors, pense quatre bouteilles pour la, pour la classe, $<$ DS: Pour trente-cinq $>$ alors hein, je t'expliques pas là. Et dans un autre coin, on a bien mangé, mais on a mangé à l'eau. Il y avait pas de vin. Pas de vin du tout. Alors à l'eau. Alors euh. Avec la classe, je t'explique pas. Il y en a quelques-uns euh (rires), enfin on a ben fait, on a ben bu avec lui. Et puis euh.

E2: Et le pain? Il y en a?

\footnotetext{
${ }^{8}$ Ce chiffre n'inclut pas les occurrences quasi-grammaticalisées de «X, c'est...' et «moi, je...'
} Si on ajoute ces occurrences à l'ensemble annoté, cela donne le total de 507 occurrences. 
GS: Oui, il y a du pain, mais euh, s/, si, il y a, il y avait un genre de pain euh, euh enfin c'était euh pff, c'est un, un pain comme, comme, pain à peu près comme chez nous, pas bien.

DS: Surtout des galettes (42ags1)

Comme on peut le voir, dans le segment précédant l'intervention du locuteur E2, il n'a pas été question du pain. Toutefois, malgré sa nouveauté discursive le référent est accessible cognitivement du fait de fonctionner sur le mode du connu emprunté au macro-topique 'la nourriture'. Dans ce sens, l'introduction d'un topique nouveau ne crée pas de rupture par rapport à la ligne thématique générale de la conversation puisque le topique n'a pas de statut de macro-topique. Les autres exemples relevés dans le corpus du français manifestent la même particularité. Ceci s'explique, à mon sens, par le fait que l'introduction d'un nouveau topique au sein de la conversation est pragmatiquement contrainte dans la mesure où le locuteur doit nécessairement tenir compte de l'état du modèle de discours qu'il co-construit avec ses interlocuteurs ainsi que des informations qui y ont déjà été intégrées lors de l'échange conversationnel. De ce fait, l'introduction de la plupart des topiques se fait au niveau local et non pas au niveau général. Ceci n’implique pas que l'introduction d'un nouveau macro-topique soit impossible à l'intérieur de la conversation. Seulement l'introduction d'un tel topique ne peut être justifié que si les interlocuteurs partagent le sentiment qu'il n'y a plus rien à dire à propos du macro-topique en cours.

Il n'en demeure pas moins que l'introduction d'un topique dans une question a pour visée de sélectionner une continuation préférentielle pour le deuxième tour, et ce indépendamment de la valeur hiérarchique du topique, ce que montre d'ailleurs l'exemple cité: les locuteurs $G S$ et $D S$ enchaînent par rapport à la question de façon explicite et cohérente sur le plan thématique.

Dans ce qui précède, nous avons examiné quelques particularités de l'utilisation de la CDG dans la paire adjacente de deux tours de parole. Il a été postulé notamment que, lorsque l'utilisation de la CDG est associée à la construction du topique par élicitation, elle tend à apparaitre, soit au premier tour de parole de la paire adjacente, soit au début du deuxième tour de parole. La spécificité de l'utilisation de la CDG dans l'alternance des tours de parole vient principalement de la spécificité d'une position séquentielle particulière. Employée au début du premier tour de la paire adjacente, plus précisément dans une question, la CDG sert à annoncer l'introduction ou la réintroduction d'un topique, tandis qu'au début du deuxième tour, elle sert à indiquer que le référent de la question est identifié et accepté en tant que topique pour le développement ultérieur.

\subsection{La CDG et la construction du topique par narration}

Dans la présente section, je m'intéresserai aux particularités de l'utilisation de la CDG dans les séquences de la conversation s'organisant selon le schéma de 
narration. Il est à noter que les séquences narratives, bien qu'homogènes du point de vue de la configuration interactionnelle, n'en présentent pas moins une organisation interne assez complexe. En se basant sur les données d'usage, Chafe (1994: 128-132) a observé que la construction du topique dans un schéma narratif inclut dans la plupart des cas les étapes suivantes:

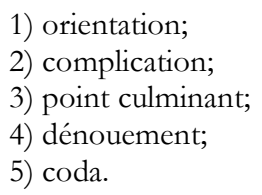

La fonction d'orientation pourrait être rapprochée du cadrage spatiotemporel. Selon Chafe (1994: 128-129), l'interprétation de l'information en rapport avec les coordonnées spatio-temporelles est cognitivement motivée dans la mesure où le cerveau a besoin, pour son bon fonctionnement, de repères qui lui permettent de localiser la plupart des topiques dans l'espace et le temps. Précisons à ce propos qu'à l'oral, le cadrage spatio-temporel prend rarement la forme des expressions précises comme dans la nuit du 1 au 2 février ou Mardi, le 7 février 2008. D'ailleurs, ce type d'information n'est pas fourni pour chaque nouvel épisode, surtout si les épisodes sont liés pragmatiquement.

La complication sert à introduire des référents, des événements et des états de choses qui préparent en quelque sorte l'approche du point culminant de l'épisode. Outre l'identité des personnages qui ont un rôle à jouer dans l'événement en préparation, le locuteur peut mentionner l'événement ou les événements d'arrière-fond.

Le point culminant a pour visée de révéler un événement inattendu vers lequel sont orientés pragmatiquement les éléments d'information fournis dans le contexte immédiatement précédent.

Pour ce qui est du dénouement, il se présente sous forme de réactions des interlocuteurs au récit du narrateur. Chafe souligne à ce propos que les réactions des interlocuteurs servent à conforter le narrateur dans son idée que l'événement culminant de son récit devait créer une surprise et que l'épisode valait donc la peine d'être relaté (1994: 131).

La coda permet au narrateur d'ajouter un commentaire métadiscursif qui clôt l'épisode. De manière générale, le locuteur revient sur l'événement culminant pour dire ce qu'il en pense ou comment il le perçoit.

Il convient de préciser que le schéma présenté ci-dessus ne se rencontre pas nécessairement tel quel dans les séquences narratives dans la mesure où certaines étapes peuvent en être absentes ou apparaittre dans une position autre que celle définie dans le schéma de base, ceci concerne notamment la coda. D'ailleurs, le premier constat que l'on peut faire en analysant les données PFC est que les séquences basées sur le schéma narratif se rencontrent principalement à l'intérieur du tour de parole. Précisons à ce sujet que l'utilisation de la CDG à l'intérieur du tour de parole n'implique pas qu'il fasse toujours partie du schéma narratif. Ceci concerne notamment les énoncés dans 
lesquels le référent introduit en position détachée dénote un concept qui n'a pas besoin de spécification spatio-temporel, lorsqu'il fait partie, par exemple, d'une définition ou d'une série d'affirmations exprimant une opinion sur un sujet quelconque. Par exemple:

(13) CP: Le développement durable c'est quoi? C'est en fait permettre le développement global au sens enfin, à la fois économique euh [...] environnemental euh, les liens sociaux euh, les projets de développement. Et $\underline{\underline{e}}$ développement durable ça doit inclure la protection de l'environnement. Mais en même temps, pas euh, ça doit pas euh, comment, enfin tu dois pas au nom de l'environnement créer à tout va des sanctuaires de la nature quoi, et où il y a plus d'activité économique parce que tu protèges l'environnement. (21acp1g)

Ayant défini plus haut les étapes principales de la narration à l'oral spontané, je propose de passer maintenant à l'étude des occurrences de la CDG lorsque celles-ci apparaissent dans un contexte narratif. Les questions qui sous-tendent la présente démarche sont les suivantes: 1) Dans quelles étapes du schéma narratif la CDG est à même d'apparaitre? 2) Quel est le 'sort' du topique introduit au sein de ce schéma? 3) Le développement du topique se fait-t-il dans les limites d'un seul épisode ou peut-il continuer au-delà de l'épisode où il a été introduit?

En partant du principe que la fonction principale de la CDG est d'annoncer l'introduction et/ou le changement du topique, on pourrait admettre que, dans la séquence narrative, la CDG est utilisée au moment de la complication au sens où, si le locuteur introduit un référent qui a un rôle à jouer dans l'événement culminant, il le fait à ce stade-là. Qu'en est-il en réalité?

Ce postulat ne se confirme que partiellement. On trouve en effet des séquences narratives dans lesquelles la CDG apparait avant le point culminant du récit comme en (14), mais ce n'est pas la seule phase où la CDG peut apparaitre:

(14) CD: Je me souviens aussi, j'avais dix-huit ans, oh oui, largement. Euh. Des amis euh, qui étaient fiancés tous les deux, ils m'ont dit 'Oh ben, on va demander à ta maman pour aller au cinéma ce soir.' (bruit de porte) Ben maman a jamais voulu. Elle a dit 'Comment? Mais il a pas assez de sa fiancée, il faut qu'il vienne te chercher?' Oh, bonté, ouh là là. Là c'était un petit peu dur hein. (42acd1g)

En (14), la locutrice commence par préciser son âge au moment des faits: ‘j'avais dix-huit ans'. Le lieu n’est pas spécifié car le présent épisode se déroule dans le même espace que les épisodes précédents. La partie complication est constituée ici par le segment suivant: 'Des amis euh, qui étaient fiancés tous les deux, ils m'ont dit "Oh ben, on va demander à ta maman pour aller au cinéma ce soir". La partie en question noue l'intrigue et prépare l'interlocuteur vers le point culminant du récit: 'Ben maman a jamais voulu. Elle a dit "Comment? Mais il a pas assez de sa fiancée, il faut qu'il vienne te chercher?". La phase de dénouement n'est pas exprimée explicitement puisque le deuxième locuteur ne se signale pas. Cependant, on pourrait supposer que la locutrice ait eu la 
possibilité d'être informée de la réaction de son allocutaire par des signes phatiques non verbaux, par exemple: un regard, un sourire, un signe de tête, etc. En revanche, la dernière phase coda est ici présente: 'Là c'était un petit peu dur hein'.

Comme on peut le constater, la CDG apparait dans la partie complication. Toutefois, le référent qu'elle sert à introduire peut jouer un rôle secondaire par rapport aux référents qui jouent un rôle principal dans l'épisode, dans le cas présent, la locutrice elle-même et sa mère. Ceci n'implique pas que le rôle du topique secondaire n'est pas important, bien au contraire. En (14), l'action en préparation dans laquelle est impliqué le référent 'les amis de la locutrice $C D$ ' contribue à la mise en relief du problème éternel qui oppose les enfants aux parents. Il n'en demeure pas moins que l'introduction du référent 'les amis de la locutrice $C D^{\prime}$ ne donne pas lieu à un vrai développement topical au sens conversationnel du terme. Au niveau formel, les expressions pronominales ils et on sont les seules expressions qui y renvoient.

Considérons maintenant le cas où l'introduction d'un topique est associée à la phase de culmination:

(15) CD: Puis on est arrivé euh en Italie, c'était, huit heures du matin, à peu près. Eh bien, en arrivant là-bas, eh ben (rires), on est allé chercher un café pour euh déjeuner. Mais en Italie, c'est qu'on s'assoit pas pour déjeuner, c'est tout au, au bar. Oh ben, on a dit, 'Non, on vient de passer une nuit, on veut au moins un petit-déjeuner confortable (rires), s'asseoir.' Ben, on a eu de la peine à trouver un espèce de petit recoin au fond de, d'une salle. Ils nous ont servi un café, alors moi, pas du tout à la française, alors le café, hum, pas digéré du tout ça.

$\mathrm{E} 2: \mathrm{Tu}$ as pas bu le cappuccino?

CD: Non, ben je le bois pas moi non. Non, je bois pas tellement du café, alors làbas, j'ai fait exception, mais j'ai demandé beaucoup d'eau. Sinon les autres ont bu le cappuccino, ben ça va de soi, hein. (42acd1)

Orientation: 'CD: Puis on est arrivé euh en Italie, c'était, huit heures du matin, à peu près'

Complication: 'CD: Eh bien, en arrivant là-bas, eh ben (rires), on est allé chercher un café pour euh déjeuner. Mais en Italie, c'est qu'on s'assoit pas pour déjeuner, c'est tout au, au bar. Oh ben, on a dit, 'Non, on vient de passer une nuit, on veut au moins un petitdéjeuner confortable (rires), s'asseoir.'

Culmination:

' $\mathrm{CD}$ : Ben, on a eu de la peine à trouver un espèce de petit recoin au fond de, d'une salle. Ils nous ont servi un café, alors moi, pas du tout à la française, alors le café, hum, pas digéré du tout ça.'

\section{Dénouement:}

'E2: Tu as pas bu le cappuccino?'

Coda: 'CD: Non, ben je le bois pas moi non. Non, je bois pas tellement du café, alors là-bas, j'ai fait exception, mais j'ai demandé beaucoup d'eau. Sinon les autres ont bu le cappuccino, ben ça va de soi, hein.' 
Notons que la CDG ne sert pas ici à annoncer l'introduction d'un topique car le référent du SN détaché le café a déjà fait l'objet de la mention antérieure [ont servi] un café. Ainsi, dans le cas présent, la fonction de la CDG se rapproche de celle que l'on attribue généralement à la CDG au début du deuxième tour de la paire adjacente en ce qu'elle permet de confirmer le centre d'intérêt en construction.

Comme on peut le constater, l'introduction du topique en association avec le point culminant de l'épisode peut jouer un rôle macro-structurant pour l'organisation des tours de parole suivants. En effet, la locutrice E2 réagit à ce qu'elle vient d'entendre au sens où la question qu'elle pose s'enchaîne aux derniers dires de la locutrice $C D$. Le tour suivant fournit d'abord une information explicative préférentiellement sélectionnée par la question et glisse ensuite vers un commentaire métadiscursif sur ce qui constitue l'enjeu du récit de $C D$, à savoir 'le café'.

\section{CONCLUSIONS}

Dans ce qui précède, nous avons vu que la CDG ne sert pas nécessairement à introduire un topique nouveau ou réintroduire un topique désactivé puisqu'elle sert également à confirmer le statut de topique à venir du référent introduit au premier tour de la paire adjacente de deux tours de parole ou à signaler l'intérêt pour le choix thématique de son interlocuteur lorsque le référent visé fonctionne déjà comme topique.

Le fait que la CDG sert à reprendre le référent ayant accédé au statut de topique ne remet pas en cause, me semble-t-il, l'analyse en termes du marquage de la relation topique/commentaire car le référent placé en position détachée à gauche joue bel et bien le rôle de topique, une fois qu'il est repris par un élément de rappel à l'intérieur de la proposition (Lambrecht, 1994: 223; auteur, 2008: 109).

Il n'en demeure pas moins que l'approche purement informationnelle de la CDG ne permet pas une interprétation satisfaisante des données dans la mesure où elle néglige quelque peu l'apport interactif de la CDG dans l'échange conversationnel (cf. De Fornel, 1988; Pekarek Doehler, 2001). Or, cet apport s'avère important pour peu que l'on mette en relation l'utilisation de la CDG avec la configuration séquentielle de la conversation.

L'étude des CDG dans leurs contextes interactifs a en effet montré que l'interprétation de la CDG est indissociable du contexte interactif dans lequel elle est utilisée. On postule notamment que la persistance topicale du référent introduit dans une CDG ne dépend pas que de son statut informationnel, comme le laissent entendre Gregory et Michaelis (2001: 1692). En effet, la durée de l'activation du topique dépend davantage des contraintes d'ordre interactionnel, à savoir: la position séquentielle, la stratégie discursive sur laquelle repose la construction du topique (élicitation, narration), la phase à laquelle est associée l'introduction du référent (cf. complication vs. 
culmination), le statut hiérarchique du topique par rapport aux autres topiques, l'intérêt que le topique suscite chez les interlocuteurs (ce dernier point concerne notamment la construction du topique par élicitation).

La prise en compte des dynamiques de l'interaction conversationnelle ne signifie pas pourtant l'abandon de l'analyse informationnelle du discours au profit de l'analyse interactive. Une hypothèse importante à ce sujet est que le niveau informationnel et le niveau interactionnel relèvent de dimensions interdépendantes et doivent en tant que telles être traitées dans une perspective qui reconnaisse 'la richesse complémentaire que peuvent apporter, d'une part, une investigation - plus fonctionnaliste ou discursive peut-être - sur la structure informationnelle du discours et, d'autre part, une interrogation - plus interactionniste - sur son organisation interactive' (Pekarek Doehler, 2001: 190).

Adresse pour correspondance:

Laboratoire Cognition, Langues, Langage, Ergonomie

Equipe de Recherche en Syntaxe et Sémantique

Maison de la Recherche

Université de Toulouse Le Mirail

5 Allées Antonio Machado

F-31058 Toulouse cedex

France

e-mail:LLidia.Pbilippe@,univ-tlse2.fr

\section{REFERENCES}

Ashby, W. (1988). The Syntax, Pragmatics, and Socio-linguistics of Left- and Right-dislocation in French. Lingua, 75: 203-229.

Auteur. (2008). Thèse de doctorat. Université de Toulouse Le Mirail.

Barnes, B. K. (1985). The Pragmatics of Left Detachment in Spoken Standard French. Amsterdam/Philadelphia: John Benjamins.

Berthoud, A.-C. (1996). Paroles à propos: approche énonciative et interactive du topic. Paris: Éditions Ophrys.

Chafe W. L. (1994). Discourse, Consciousness, and Time. Chicago and London: The University of Chicago Press.

Charolles, M. (2002). La référence et les expressions référentielles en français. Paris: Ophrys.

Dik, S. C. (1997). The Theory of Functional Grammar: Complex and derived constructions, $2^{\text {nd }}$ edn, part 2. Berlin/New York: Mouton de Gruyter.

Durand, J., Lyche Ch. et Laks, B. (2002). Protocole d'enquête. Bulletin PFC, 1: 5-19. Editions ERSS. 
Durand, J. et Lyche, Ch. (2003). Le projet Phonologie du français contemporain et sa méthodologie. In: J. Durand et E. Delais-Roussarie (eds), Corpus et variation en phonologie du français: méthodes et analyses, Toulouse: Presses Universitaires du Mirail, pp. 213-276.

Durand, J. et Tarrier, J.-M. (2006). PFC, corpus et systèmes de transcription. Cabiers de Grammaire, 30: 139-158, Editions ERSS.

Fornel (de), M. (1988). Constructions disloquées, mouvement thématique et organisation préférentielle de la conversation. Langue Française, 78: 101123.

Geluykens, R. (1992). From Discourse Process to Grammatical Construction: on LeftDislocation in English. Amsterdam: John Benjamins.

Gramley, S. and Pätzold, K.-M. (2003). A Survey of Modern English. London/ New York: Routledge.

Gregory, M.L. and Michaelis, L.A. (2001). Topicalization and Left-dislocation: A Functional Opposition Revisited. Journal of Pragmatics, 33(11): 16661706.

Grice, H. P. (1975). Logic and Conversation. In: P. Cole and J. L. Morgan (eds), Syntax and Semantics, vol. 3: Speech Acts. New York: Academic Press, pp. 41-58.

Grobet, A. (2002). L'identification des topiques dans les dialogues, Bruxelles: Editions Duculot.

Gundel, J. K. (1988a). Universals of Topic-Comment Structure. In: M. Hammond, E. Moravcsik and J. Wirth (eds), Studies in Linguistic Typology. Amsterdam, Philadelphia: John Benjamins, pp. 209-239.

Gundel, J. K. (1988b). The Role of Topic and Comment in Linguistic Theory, New York and London: Garland Publishing, Inc.

Gundel, J. K., Hedberg, N. and Zacharski, R. (1993). Cognitive Status and the Form of Referring Expressions in Discourse. Language, 69 (2): 274-307.

Lacheret, A. et François, J. (2003). De la notion de détachement topical à celle de constituant thématique extrapositionnel. Cabiers de praxématique, 40: 167-198.

Lambrecht, K. (1981). Topic, Antitopic and Verb Agreement in Non-standard French. Amsterdam: John Benjamins.

Lambrecht, K. (1994). Information structure and sentence form: Topic, focus, and the mental representations of discourse referents. Cambridge: Cambridge University Press.

Lambrecht, K. (2001). Dislocation. In: M. Haspelmath, E. König, W. Oesterreicher and W. Raible (eds), Language Typology and Language Universals, Berlin/NewYork: de Gruyter, pp. 1050-1078.

Larsson, E. (1979). La dislocation en français: Etude de syntaxe générative. Etudes Romanes de Lund 28, Sweden: Lund.

Morel, M.-A. (1989). Structure interne des énoncés comportant une question: topique-question. In: M.-A. Morel (ed.), Analyse linguistique d'un corpus, Paris: Publications de la Sorbonne Nouvelle, pp. 151-167. 
Pekarek Doehler, S. (2001). Dislocation à gauche et organisation interactionnelle. Marges linguistiques, 2: 177-194.

Prévost, S., 2003, Détachement et Topicalisation: des niveaux d'analyse différents. Cahiers de praxématique, 40: 97-126.

Prince, E. F. (1981). Toward a Taxonomy of Given-New Information. In: P. Cole (ed.), Radical Pragmatics, NY: Academic Press, pp. 223-56.

Prince, E. F. (1997). On the Functions of Left-Dislocation in English Discourse. In Kamio A. (ed.), Directions in functional linguistics. Philadelphia, PA: John Benjamins, pp. 117-143. Article on line: http://www.ling.upenn.edu/ ellen/home.html

Roulet, E., L. Filliettaz et Grobet, A. (2001). Un modèle et un instrument d'analyse de l'organisation du discours. Bern: Peter Lang SA.

Sacks, H. (1987). On the Preference of Agreement and Continuity in Sequences in Conversation. In: G. Button and J.R. Lee (eds), Talk and Social Organization. Clevedon: Multilingual Matters, pp. 54-59.

Ziv, Y. (1994). Left and Right Dislocations: Discourse Functions and Anaphora. Journal of Pragmatics, 22: 629-645. 